\title{
Potential Control of Oxygen Non-Stoichiometry in Cerium Oxide and Phase Transition Away from Equilibrium
}

Catherine Dejoie, ${ }^{\text {I }}$ Yi Yu, ${ }^{\text {If }}$ Fabiano Bernardi, Nobumichi Tamura, Martin Kunz, Matthew A. Marcus, Yi-Lin Huang, Chunjuan Zhang, Bryan W. Eichhorn,* and Zhi Liu*

Cite This: ACS Appl. Mater. Interfaces 2020, 12, 31514-31521

Read Online

ABSTRACT: Cerium oxide (ceria, $\mathrm{CeO}_{2}$ ) is a technologically important material for energy conversion applications. Its activities strongly depend on redox states and oxygen vacancy concentration. Understanding the functionality of chemical active species and behavior of oxygen vacancy during operation, especially in hightemperature solid-state electrochemical cells, is the key to advance future material design. Herein, the structure evolution of ceria is spatially resolved using bulksensitive operando X-ray diffraction and spectroscopy techniques. During water electrolysis, ceria undergoes reduction, and its oxygen non-stoichiometry shows a dependence on the electrochemical current. Cerium local bonding environments vary

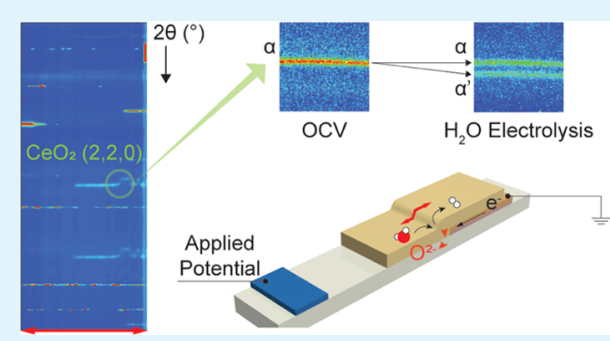
concurrently to accommodate oxygen vacancy formation, resulting in changes in $\mathrm{Ce}-\mathrm{O}$ coordination number and $\mathrm{Ce}^{3+} / \mathrm{Ce}^{4+} \mathrm{redox}$ couple. When reduced enough, a crystallographic phase transition occurs from $\alpha$ to an $\alpha^{\prime}$ phase with more oxygen vacancies. Nevertheless, the transition behavior is intriguingly different from the one predicted in the standard phase diagram of ceria. This paper demonstrates a feasible means to control oxygen non-stoichiometry in ceria via electrochemical potential. It also sheds light on the mechanism of phase transitions induced by electrochemical potential. For electrochemical systems, effects from a large-scale electrical environment should be taken into consideration, besides effective oxygen partial pressure and temperature.

KEYWORDS: ceria, oxygen vacancy, phase transition, operando, X-ray diffraction

\section{INTRODUCTION}

Cerium oxide (ceria, $\mathrm{CeO}_{2}$ ) is a technologically important and fundamentally interesting material because of its highly tunable redox chemical properties. It has been widely used in three-way catalysts, ${ }^{1,2}$ energy conversion devices, ${ }^{3-6}$ oxygen permeation membranes, ${ }^{7}$ and also in biotechnology and medicine. ${ }^{8,9}$ These applications are mostly related to the rapid formation/ annihilation of oxygen vacancy $\left(\mathrm{V}_{\mathrm{O}}^{\bullet \bullet}\right)$ and the strong impact of oxygen vacancy on the chemical and physical properties of ceria. ${ }^{10}$ Therefore, the ability to monitor, modify, and precisely control oxygen vacancy is a key prerequisite for being able to control ceria properties and design ceria-based materials in a rational manner.

Ceria has a robust fluorite structure that can accommodate a large oxygen non-stoichiometry over a wide temperature and oxygen partial pressure range as $\mathrm{CeO}_{2-x^{*}}{ }^{10,11}$ This feature enables the storage and supply of oxygen in ceria and ceriabased materials, which is important for catalytic applications. ${ }^{2,12}$ Upon oxygen release, the reduction of $\mathrm{Ce}^{4+}$ to $\mathrm{Ce}^{3+}$ and the incorporation of oxygen vacancies in its fluorite-type structure result in a volume expansion of the material. ${ }^{13}$ This is well known as chemical expansion, ${ }^{13}$ with a linear relationship between the cell parameter and oxygen non-stoichiometry $x$. This phenomenon presents a problem in mechanical engineering because of induced material strain and structural deflection. However, on the other hand, it is of particular interest for mechanical sensing and actuation applications. ${ }^{14}$ For instance, one could monitor oxygen partial pressure from expansion/ contraction of the structure; one could also control such oxide expansion/contraction for material actuation either by electrical tuning of oxygen vacancy concentration or by adjusting the gas atmosphere. Recently, Swallow et al. ${ }^{14}$ reported "breathing" response of a model material $\mathrm{Pr}_{x} \mathrm{Ce}_{1-x} \mathrm{O}_{2-\delta}$ with strain $>0.1 \%$ through electrochemical pumping of oxygen with electrical potential $<0.1 \mathrm{~V}$. This work noted a mechanism that couples electrical stimulus to mechanical properties via material defect chemistry, which leads to a new design path for electromechanical actuation with non-stoichiometric oxide films. In a recent review, $\mathrm{Li}$ and Chueh $^{15}$ discussed an ion insertion process by chemical and electrochemical stimuli in many solid oxides. In addition to this, change in oxygen non-stoichiometry results also in distinct electrical properties ${ }^{15-18}$ and more fundamentally in phase transition, leading to a complex phase diagram.

Received: May 6, 2020

Accepted: June 19, 2020

Published: June 19, 2020 
a)

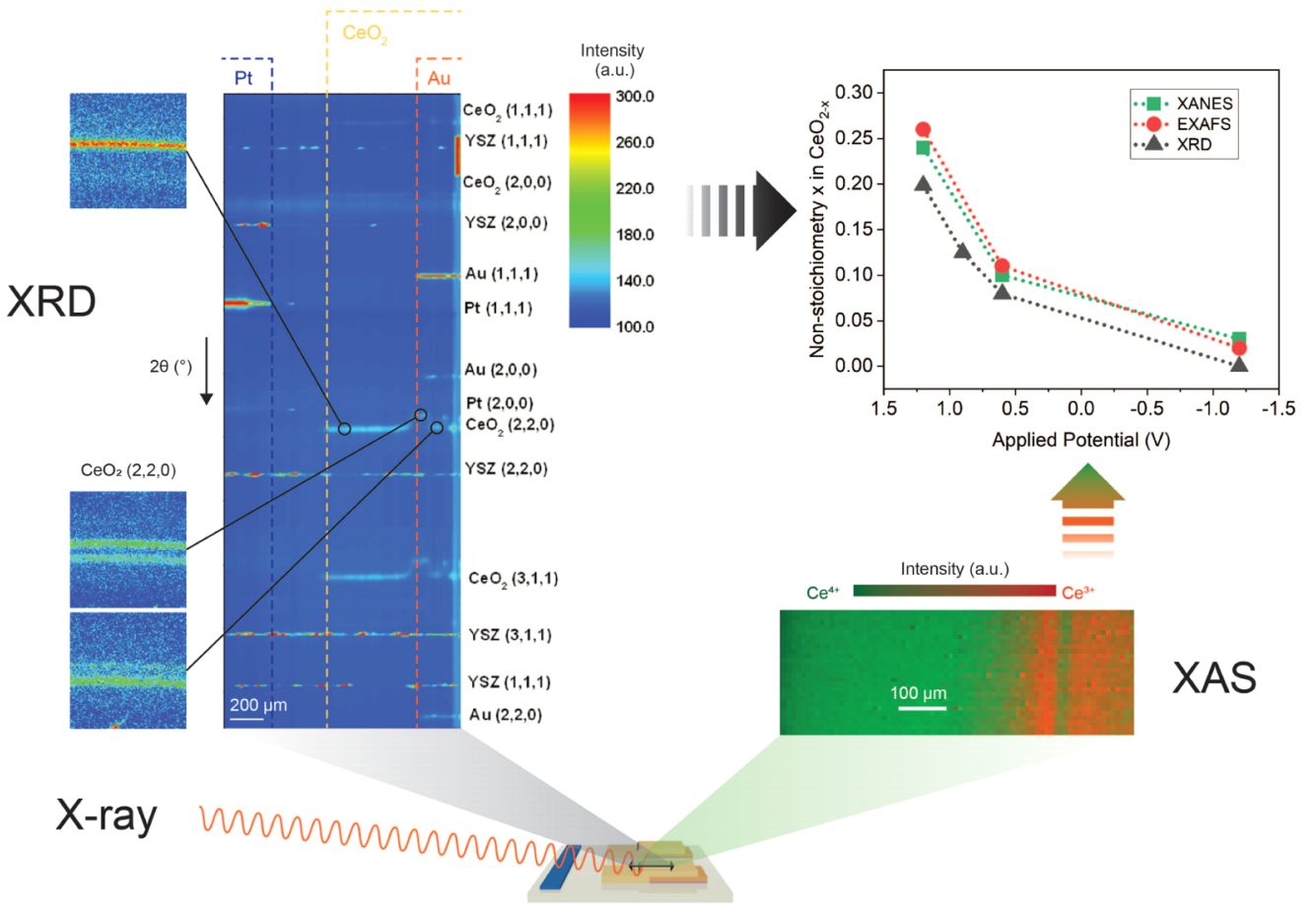

b)

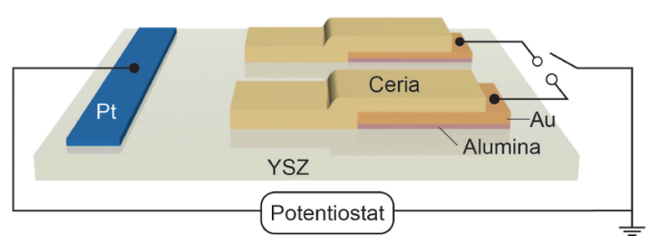

c)

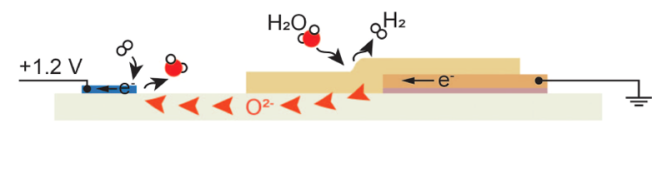

Figure 1. Operando behaviors of ceria are revealed by a suite of synchrotron-based X-ray techniques. (a) XRD and XAS are employed to study the bulk properties of a ceria electrode in a model SOC. XRD patterns are shown along a line crossing the SOC under the steady state of operation. Diffraction reflections of $\mathrm{CeO}_{2}, \mathrm{Au}$, and $\mathrm{YSZ}$ are indicated. XAS mapping is obtained in fluorescence mode. Intensity of the contour plot represents the relative concentration of $\mathrm{Ce}^{3+}$ (red) and $\mathrm{Ce}^{4+}$ (green). Figure inset gives the extracted $x$ values from XRD, X-ray absorption near edge structure, and extended X-ray absorption fine structure as a function of potential at $611{ }^{\circ} \mathrm{C}$. (b) SOC consists of a $300 \mathrm{~nm} \mathrm{Pt} \mathrm{CE} \mathrm{(blue),} 300 \mathrm{~nm} \mathrm{Au} \mathrm{pad}$ current collectors (orange) on top of $30 \mathrm{~nm}$ alumina films (red), and $1000 \mathrm{~nm}$ ceria WEs (yellow) patterned onto a polycrystalline YSZ substrate (light grey). During operation, the ceria WEs are grounded and negatively biased relative to the Pt CE. This schematic drawing is not to scale. (c) SOC is heated above $500{ }^{\circ} \mathrm{C}$ in a gas mixture of $\mathrm{H}_{2}$ and $\mathrm{H}_{2} \mathrm{O}$. The negative potential on ceria drives $\mathrm{H}_{2} \mathrm{O}$ electrolysis on the ceria surface, while the positive potential promotes $\mathrm{H}_{2}$ electro-oxidation at the Pt side.

With a suite of synchrotron-based X-ray techniques, we aim to gain a more comprehensive picture of oxygen vacancy behavior from the surface through the bulk structure and verify the mechanism of potential-induced phase transition by using ceria as a model system. Such fundamental understanding is crucial for heterogeneous catalytic processes and especially for actuation applications where it is desirable to control the material properties by applied potentials. Our previous ambient pressure X-ray photoelectron spectroscopy (APXPS) studies have revealed an extended electrochemical surface active region beyond the three-phase boundary. ${ }^{19,20}$ In this paper, we probe the ceria electrode from the surface to the bulk. An external potential was applied to a solid oxide electrochemical cell (SOC) consisting of a ceria film as the working electrode (WE), an yttria-stabilized zirconia (YSZ) electrolyte, and a platinum counter electrode (CE). Our model SOC is designed such that the oxygen vacancy (and ion) transport is confined within a certain region, allowing one to discern changes in structure and vacancy concentration as a function of current and temperature. ${ }^{19-22}$ We used operando $\mathrm{X}$-ray diffraction (XRD) and X-ray absorption spectroscopy (XAS) (Figure 1a) to examine the structural properties of ceria, with gas pressure and temperature systematically controlled. Probing the vacancy distribution through ceria bulk, we obtained a direct correlation of oxygen vacancy concentration with the electrochemical current. In addition, our results clearly show that the non-stoichiometry $x$ in $\mathrm{CeO}_{2-x}$ and associated phase transition in the relevant nonstoichiometry range can be tuned by controlling the electrical potential.

This work first demonstrates control of non-stoichiometry through electrochemical potential in functional oxides with a $\mathrm{CeO}_{2-x}$ model system. This electrochemical method is an easily accessible and practical means to tune electrical and mechanical properties of solid oxide materials. It also provides new possibility in heterogeneous catalysis, especially at elevated temperatures. Beyond changes in temperature, it adds an extra dimension to introduce/remove particular catalytic active sites by controlling the amount of oxygen vacancies. Second, this work also reveals a detailed mechanistic understanding of the phase change induced by electrochemical potential. It has been reported that the effective oxygen partial pressure as changed by electrical stimulus has the same effect on the phase transition induced by the actual change of oxygen 
a)

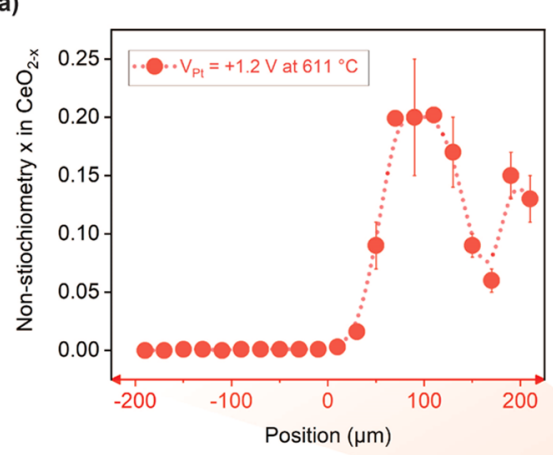

b)

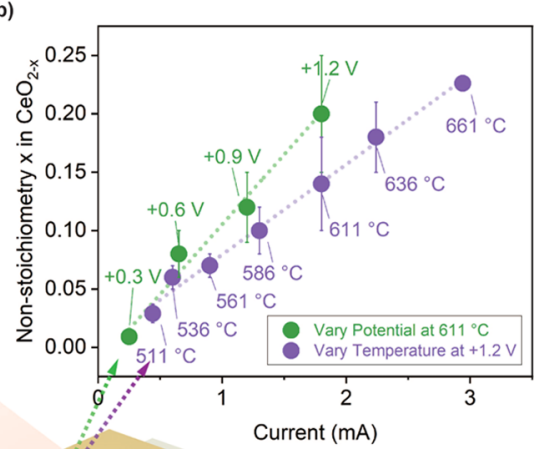

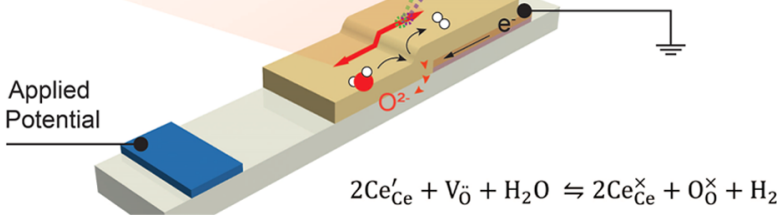

Figure 2. Ceria shows bulk reduction during operation, and the oxygen non-stoichiometry $x$ has a linear dependence on cell current. (a) Oxygen non-stoichiometry $x$ on the ceria electrode across the SOC at $611^{\circ} \mathrm{C}$ WT and $+1.2 \mathrm{~V}$ applied potential on Pt. These values are extracted from XRD measurement obtained along the red line. The WT values have been calibrated, and the $+1.2 \mathrm{~V}$ applied potential denotes cathodic reaction on the ceria electrode. (b) Change in oxygen non-stoichiometry $x$ as a function of applied cell potentials (denoted in green) and as a function of temperature (denoted in purple) on the ceria electrode. The linear fits are also displayed for reference.

partial pressure, following the Nernst equation. ${ }^{23}$ However, a clear different phase transition behavior is observed in this study when compared with the standard ceria phase diagram. ${ }^{24}$ New information is provided from this investigation to clarify the phase transition mechanism via potential control. Finally, there is a general implication on the relevance of ceria and similar fluorite-structure oxides for energy conversion and storage applications. Under realistic non-open circuit voltage conditions, a large deviation in the structure and the chemical state can be induced by a finite change in electrical potential. The electrochemical activity, ionic and electronic transport properties, and stability of these oxide materials can be largely altered as a consequence. Therefore, it is important to assess such changes under in situ and operando environments and to identify the threshold potential that leads to phase transitions.

\section{RESULTS AND DISCUSSION}

Operating the Ceria-Based SOC. To perform simultaneous electrochemical evaluation and X-ray powder diffraction/absorption experiments, we employed a specially designed model SOC. The single-sided SOC consists of polycrystalline YSZ electrolyte, ceria WEs with Au pad current collectors, and Pt CE (Figure 1b). All the cell components are positioned on the same side to facilitate in situ X-ray measurements. Dense ceria films (1000 nm-thick) are deposited on top of $\mathrm{Au}$ pads in a way that only the ceria edge close to the Pt CE has direct contact with the YSZ electrolyte. Underneath the Au pads, insulating $30 \mathrm{~nm}$ thick alumina layers are deposited to block direct ionic transport between the YSZ electrolyte and the Au current collectors, thus allowing well-defined current flow regions. A detailed description of the cell geometry can be found in previous publications. $^{19,20}$

The SOC was mounted on top of an Anton Paar DHS900 heater and heated to a particular working temperature (WT) (from 510 to $665{ }^{\circ} \mathrm{C}$ after calibration, see Supporting Information for details in temperature calibration) under a 40 Torr of $1: 1 \mathrm{H}_{2}$ and $\mathrm{H}_{2} \mathrm{O}$ mixture (Figure $\mathrm{S} 1$ ). At open circuit voltage $(\mathrm{OCV})$, the ceria surface reaches thermal equilibrium with $\mathrm{H}_{2}$ and $\mathrm{H}_{2} \mathrm{O}$ in the gas phase, as described by the following equation

$$
2 \mathrm{Ce}_{\mathrm{Ce}}^{\prime}+\mathrm{V}_{\mathrm{O}}^{\bullet \bullet}+\mathrm{H}_{2} \mathrm{O} \leftrightharpoons 2 \mathrm{Ce}_{\mathrm{Ce}}^{\times}+\mathrm{O}_{\mathrm{O}}^{\times}+\mathrm{H}_{2}
$$

where $\mathrm{Ce}_{\mathrm{Ce}}^{\prime}, \mathrm{Ce}_{\mathrm{Ce}}^{\times}, \mathrm{V}_{\mathrm{O}}^{\bullet \bullet}$, and $\mathrm{O}_{\mathrm{O}}^{\times}$are $\mathrm{Ce}^{3+}, \mathrm{Ce}^{4+}$, doubly positive charged oxygen vacancy, and oxide ion on oxygen site, respectively. With no net current flowing through the SOC, this equilibrium is determined by temperature and gas partial pressures.

If a potential is applied between the ceria WE and the Pt CE, charged species move through the cell as a current flow, driving the surface redox states and coverage away from thermal equilibrium and promoting the surface chemistry in eq 1 . With the $\mathrm{Au}$ current collector grounded, the cell potential is controlled by the applied potential on the Pt CE through a potentiostat: $V_{\text {cell }}=V_{\mathrm{Pt}}-V_{\mathrm{Au}}$. For example, when the $\mathrm{Pt}$ electrode is under positive potential (e.g., $+1.2 \mathrm{~V}$ in Figure 1c), electrons move from the $\mathrm{Au}$ current collector to the ceria electrode, while oxide ions are driven from the ceria film to the YSZ electrolyte and are conducted to the Pt CE. Therefore, the forward reaction of eq $1, \mathrm{H}_{2} \mathrm{O}$ electrolysis, is favored on ceria. If $\mathrm{Pt}$ is negatively biased, the fluxes of $\mathrm{O}^{2-}$ and polarons are switched, leading to $\mathrm{H}_{2}$ electro-oxidation (eq 1 , reverse reaction) on the ceria surface and $\mathrm{H}_{2} \mathrm{O}$ electrolysis at the $\mathrm{Pt}$ electrode. The current-voltage profile that reflects the activity of the SOC is given in Figure S2. Note that during electrochemical reactions, the local region of each cell component is subject to different "real" potential depending on ohmic loss and reaction kinetics. ${ }^{19-21}$ Hence, only the cell applied potential of +1.2 and $-1.2 \mathrm{~V}$ are given to denote cathodic and anodic reactions on the ceria electrode, respectively.

Bulk Reduction of Ceria during Water Electrolysis. The accepted view on ceria reduction/oxidation behavior suggests several differences between the surface and the bulk. In general, the ceria surface is more enhanced with reduced states compared with the bulk over wide ranges of temperature 
and oxygen partial pressure. ${ }^{25-28}$ Its redox dependence on the environment could exhibit another stark contrast. ${ }^{29}$ Ceria redox behavior can continually evolve when it is driven further away from equilibria. In our previous APXPS studies, we have shown that ceria is surface-reduced upon cathodic potential, as signaled by shift in $\mathrm{Ce}^{3+} / \mathrm{Ce}^{4+}$ ratio. ${ }^{19-21}$ Herein, we find that ceria is also reduced through the bulk by using XRD and XAS. In Figure 1a, the XRD peaks of ceria shift toward smaller $2 \theta$ values during operation, consistent with chemical expansion of reduced ceria. ${ }^{13,30}$ The same reduction regions are concomitantly revealed in the $\mathrm{Ce}^{3+} / \mathrm{Ce}^{4+}$ chemical map derived from XAS measurement. The oxygen non-stoichiometry values obtained from X-ray absorption near edge structure (XANES), extended X-ray absorption fine structure (EXAFS), and XRD (inset Figure 1a) are consistent, with a slightly higher degree of reduction revealed in XANES and EXAFS measurements. This small discrepancy indicates that ceria reduction occurs through the bulk structure mainly with good crystal quality but accompanied by a small amount in the amorphous or disordered state.

Oxygen non-stoichiometry $x$ values extracted from XRD characterization across the ceria electrode bulk during operation are summarized in Figure 2. This is achieved by first extracting the ceria cell parameter using Le Bail refinement and then applying Kim's law ${ }^{31}$ (see Supporting Information for more details). Ceria is mostly oxidized under the OCV condition at $611^{\circ} \mathrm{C}$ (Table S2). Upon applied potential of $+1.2 \mathrm{~V}$, varying degrees of reduction are observed from the bulk non-stoichiometry $x$ map along the ceria electrode (Figure 2a and Table S6), with the highest $x$ values obtained close to the edge of the Au current collector. A similar pattern is delineated in our previous reports on surface redox state mapping. ${ }^{19-21}$ Such similarity reveals that reduction in the bulk is following the one on the surface. More essentially, it captures the propagation of reduction from the surface region to the bulk structure, as a result of the rate-limiting kinetics of surface reaction. ${ }^{19}$ It was noted that this reduction was observed only during cathodic potential. Ceria was oxidized back to the original OCV condition soon after the removal of applied potential, as confirmed by both XRD and XAS measurements. This also excludes possible reduction effects from the X-ray beam or by elevated temperatures.

To find possible correlation between the non-stoichiometry $x$ and other parameters, the cell current was systematically varied, either by changing the applied potential at a constant calibrated WT $\left(611^{\circ} \mathrm{C}\right)$ or by changing the WT at a constant applied potential $(+1.2 \mathrm{~V}$ on $\mathrm{Pt})$. A linear dependence of bulk oxygen non-stoichiometry $x$ on the cell current was observed (Figure 2b). This agrees with our previous finding of ratelimiting surface kinetics on ceria. ${ }^{21}$ If surface kinetics of water electrolysis is fast, we should expect the oxygen vacancies to be rapidly filled, and therefore, the linear increase of oxygen nonstoichiometry $x$ with the cell current should not be observed. The oxygen non-stoichiometry determined at $+1.2 \mathrm{~V}$ (on $\mathrm{Pt}$ ) while scanning the potential (denoted in green) is larger than, yet within the error of measurement, the one obtained at 611 ${ }^{\circ} \mathrm{C}$ during temperature scan (denoted in purple) for a given current. This is due to the difference in the measurement spot of the two scans. The EXAFS measurements also show similar dependence of bulk oxygen vacancy (i.e., non-stoichiometry) on electrical potential (Figure 1a inset and Figure S12). The linear dependence (at a given cell location) reveals that the ceria redox states can be effectively controlled by tuning electrical stimulus and/or thermal stimulus. A more fundamental correlation of ceria non-stoichiometry and the electrochemical potential is currently under investigation with different cell geometries and will be included in a future work.

Non-Equilibrium $\boldsymbol{\alpha}-\boldsymbol{\alpha}^{\prime}$ Phase Couple. In the temperature range of $450-650{ }^{\circ} \mathrm{C}$ and for oxygen non-stoichiometry $x$ of $0.01-0.16$, there is a region in the ceria phase diagram where two cubic phases of ceria coexist, denoted as $\alpha$ and $\alpha^{\prime}$ (higher oxygen-deficient phase). This is the so-called miscibility gap as depicted in the reproduced phase diagram ${ }^{32}$ in Figure 3a. At our WT of $611^{\circ} \mathrm{C}$, any deficient ceria with an

a)

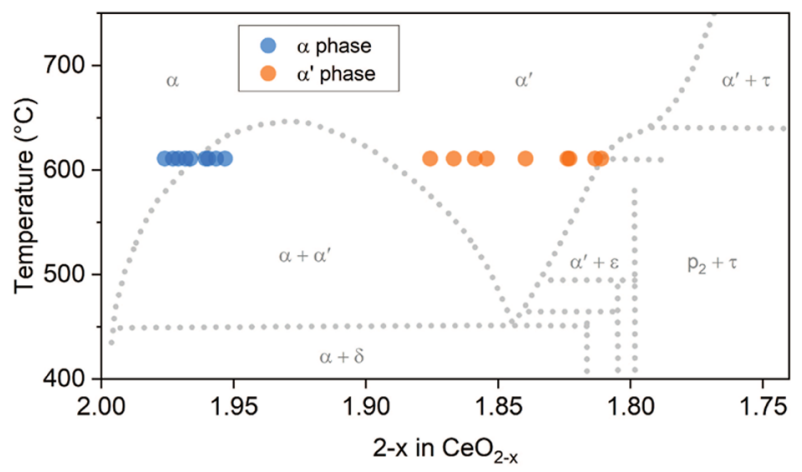

b)

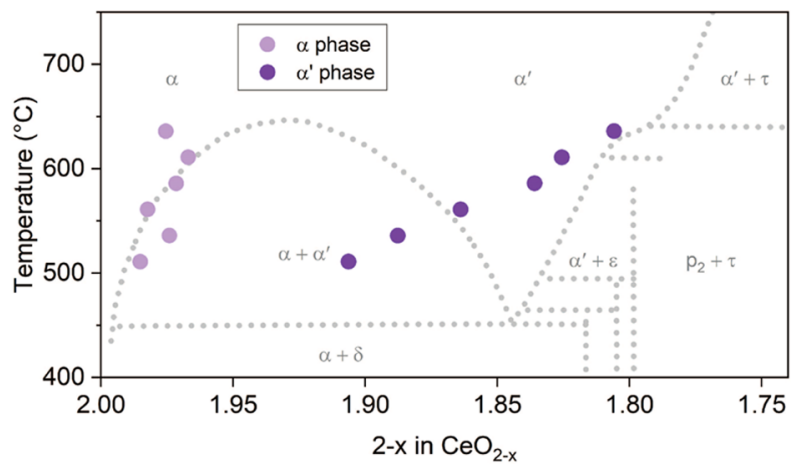

Figure 3. Ceria $\alpha-\alpha^{\prime}$ phase couple during water electrolysis. (a) Oxygen non-stoichiometry reported in the ceria phase diagram. The 2 $-x$ values are extracted from operando XRD measurements on the ceria electrode at $611{ }^{\circ} \mathrm{C}$ during $\mathrm{H}_{2} \mathrm{O}$ electrolysis. (b) XRD-derived oxygen non-stoichiometry at $+1.2 \mathrm{~V}$ under different WTs. Data are collected at a fixed position where $\alpha$ and $\alpha^{\prime}$ phases co-exist and are reported in the reproduced ceria phase diagram. ${ }^{32}$ The equilibrium phase diagram is reproduced with permission from ref 32 . Copyright 1985 Elsevier.

oxygen non-stoichiometry $x$ of $0.036-0.106$ would separate into $\alpha$ and $\alpha^{\prime}$ phases, with a composition of $\mathrm{CeO}_{1.964}$ and $\mathrm{CeO}_{1.894}$, respectively. As predicted by the lever rule, the only difference in phase separation between two deficient ceria inside the miscibility gap (e.g., $\mathrm{CeO}_{1.95}$ and $\mathrm{CeO}_{1.92}$ at $611^{\circ} \mathrm{C}$ ) lies in the relative amounts (relative intensities) of the two phases. This is illustrated by our simulation of powder diffraction patterns of ceria for several oxygen nonstoichiometries (Figure S3).

However, when the $\alpha-\alpha^{\prime}$ phase couples derived from our operando XRD measurements at $611{ }^{\circ} \mathrm{C}$ are plotted (Figure $3 \mathrm{a})$, such expected behaviors of the $\alpha-\alpha^{\prime}$ phase couples are not observed. If data points corresponding to the $\alpha$ phase mostly gather at the left boundary of the miscibility gap, those 
corresponding to the $\alpha^{\prime}$ phase exhibit an unexpected behavior as they do not cluster at the right boundary. The lowest $2-x$ value of the $\alpha^{\prime}$ phase in a $\alpha-\alpha^{\prime}$ phase couple is found at 1.811 . Again, these $\alpha-\alpha^{\prime}$ phase couples were observed only during $\mathrm{H}_{2} \mathrm{O}$ electrolysis. Ceria regained its oxidized state in the $\alpha$ phase once the cell was returned to the OCV condition. Beam effect and temperature effect are thus excluded as well.

Furthermore, we investigated these two phases by their relative intensities and coherently diffracting domain size as a function of $2-x$ (Figures S4 and S5). The $\alpha$ phase, in both its relative intensity and coherent domain size, seems to randomly scatter over a small $2-x$ range without following any obvious trend. The $\alpha^{\prime}$ phase, on the other hand, reveals a general increase in the relative intensity and domain size against the 2 $-x$ values. This observation is in relation with the splitting mechanism when going through the miscibility gap: phase transition occurs inside each nanocrystal of ceria, and both intensity and domain size of the $\alpha^{\prime}$ phase increase at the expense of the $\alpha$ phase.

We conclude that each $\alpha-\alpha^{\prime}$ phase couple seems to denote its own discrete phase diagram. Phase separation is a direct consequence of the Gibbs free energy of mixing versus the chemical composition at a given temperature. If the homogeneous solution is unstable within a composition range ( $x$ of $0.036-0.106$ at $611{ }^{\circ} \mathrm{C}$ ), the separation of the mixture into two phases ( $\alpha$ and $\left.\alpha^{\prime}\right)$ would occur and effectively reduce the Gibbs free energy. The common tangent line to the Gibbs free energy curve gives the points of the two compositions $\left(\mathrm{CeO}_{1.964}\right.$ and $\left.\mathrm{CeO}_{1.894}\right)$, which hence mark the limit of thermodynamic stability. Because the Gibbs free energy curve is a function of temperature, the thermodynamic stability limits should be fixed if the temperature is unchanged. Therefore, our results indicate that, even at the same temperature, there is a shift of free energy curves of the two phases induced by electrochemical potential. Such relative shift leads to the consequence that the chemical composition values (i.e., $2-x$ of $\alpha$ and $\alpha^{\prime}$ phases) determined from the common tangent of the free energy curves deviate from the equilibrium stability limits, even though they are obtained at the same temperature of $611{ }^{\circ} \mathrm{C}$. As we have noted above, the "real" potential differs for local regions; thus, only the cell applied potential is given for qualitative discussion herein. Precise determination of the electrochemical potential is beyond the scope of this work and will be a future focus that requires different cell geometry and experimental design.

Finally, we take a closer look at how this system is affected by the temperature parameter. On the ceria electrode where both $\alpha$ and $\alpha^{\prime}$ phase co-exist at cathodic applied potential $(+1.2 \mathrm{~V}$ on $\mathrm{Pt})$, the $\mathrm{WT}$ was systematically varied between 511 and $661{ }^{\circ} \mathrm{C}$ (as illustrated in Figure $2 \mathrm{~b}$ ). The $2-x$ values obtained at six different temperatures are reported in Figure $3 \mathrm{~b}$ in the reproduced ceria phase diagram. Similar to that in Figure $3 \mathrm{a}$, the $\alpha$ phase composition stays approximately on the left boundary of the miscibility gap, while the $\alpha^{\prime}$ phase composition deviates significantly from the right boundary. In addition, when temperature is decreased, the $\alpha^{\prime}$ phase border shifts toward more oxidized stoichiometry and eventually ends inside the classic miscibility gap. This observed shrinking of the miscibility gap indicates a relative decrease of the $\alpha^{\prime}$ phase free energy curve, compared to that of the $\alpha$ phase. Therefore, at lower temperatures, the $\alpha^{\prime}$ phase becomes energetically favorable and phase transition from $\alpha$ to $\alpha^{\prime}$ finishes at a more oxidized non-stoichiometry.
Phase Separation under Electrochemical Potentials. It was reported in literature for perovskite oxides that structural evolution and the associated phase change induced by applying electrochemical potential are equivalent to that triggered by changing the oxygen partial pressure in the environment. ${ }^{23,33,34}$ However, our findings in this study suggest some difference. On one hand, consistency is shared with previous reports as we have also verified the control of oxygen non-stoichiometry in ceria via electrochemical potential without changing the gas atmosphere. But on the other hand, we did not observe the phase change behavior typical for cases under thermal equilibrium, that is, phase change does not follow the classic phase diagram of deficient ceria. Upon electrochemical potentials, the miscibility gap right boundary for $\mathrm{CeO}_{2-x}$ at a given relatively high temperature is found to expand toward the reduced non-stoichiometry, while at lower temperatures, this boundary is observed to shrink toward the oxidized nonstoichiometry.

In order to examine the miscibility gap behavior under electrochemical potentials, we calculated the right boundary position limits. From the linear dependence of $\alpha^{\prime}$ relative intensity on $2-x$ values at $611^{\circ} \mathrm{C}$ (Figure S4), we found that phase transition occurs at the composition $\mathrm{CeO}_{1.879}$, where the $\alpha^{\prime}$ phase starts to exist and the nanocrystal turns completely into the $\alpha^{\prime}$ phase at $\mathrm{CeO}_{1.798}$. In addition, the evolution of $\alpha^{\prime}$ phase relative intensity with temperature (shown in Figure S6a) allows us to extract the temperature limits. A single $\alpha$ phase is found for temperatures below $480{ }^{\circ} \mathrm{C}$ with a chemical composition of $\mathrm{CeO}_{1.933}$, while a single $\alpha^{\prime}$ phase is obtained at temperatures above $659{ }^{\circ} \mathrm{C}$ as $\mathrm{CeO}_{1.780}$. The deduced limits (Figure S7) suggest that, compared to the thermal equilibrium condition shown in the classic phase diagram, a cathodic electrochemical potential shifts the right boundary toward more reduced stoichiometry (smaller $2-x$ value) at high temperatures and to a more oxidized state (larger $2-x$ value) at lower temperatures. Phenomenally, because only cathodic potentials are introduced in this study, such shifts are unlikely to be associated with direction of the current passage but most probably with the magnitude of it. The higher the temperature, the larger the cell current is delivered. Once it passes a threshold, the miscibility gap is switched from shrinking to expanding. More fundamentally, this observation can suggest the presence of a threshold in electrochemical potential that essentially shifts the relative free energy between $\alpha$ and $\alpha^{\prime}$ phases. An electrochemical system is special in that it requires the same magnitude in electrochemical potential of the two phases where phases separate under steady state. However, the chemical potential and electrical potential of the two phases are not necessarily the same because of their different properties. Ultimately, the electrochemical potential is determined by the coupling of oxygen vacancy concentration and the electrical driving force in the two phases. Therefore, the observed deviation in phase separation behavior from the conventional phase diagram is understandable because the latter considers free energy (i.e., chemical potential) only. Also, it is unlikely that material structure feature ${ }^{21}$ could play a role in this unexpected phase separation behavior because the ceria crystallites are small in size (Figure S5) with no preferred orientation. Note that our experimental data from the $\alpha$ phase do not allow a certain exclusion of any possible evolution of the left boundary position. Future work will focus on precise determination of the exact shape of the miscibility gap and on better understanding the mechanism behind it. We would like 
to reiterate in this work that while considering phase transition in a system subject to electrochemical potential, one should consider electrochemical free energy, which includes effects from the large-scale electrical environment, instead of chemical free energy. ${ }^{35}$ Moreover, volume change due to chemical expansion and entropy change due to oxygen vacancy incorporation upon reduction bring additional contribution to the relative shift in free energy curves of the two phases. ${ }^{15}$

\section{CONCLUSIONS}

In conclusion, the oxygen vacancy evolution in ceria and the associated phase transition were studied using operando synchrotron XRD and XAS. We found that upon cathodic potential, the ceria is reduced not only on the surface but also through the bulk. This is consistent with our previous reports of the rate limiting surface exchange reaction. ${ }^{19-21}$ Within the electrochemical active region, ceria is bulk-reduced both in crystalline structure (characterized by XRD) and in local atomic order (by XAS). Therefore, from these bulk-sensitive studies and previous surface-sensitive measurements, we obtain a full picture of the oxygen vacancy behavior in ceria operando. By using this model system of ceria, we aim to demonstrate the capabilities of in situ/operando X-ray synchrotron-based techniques in material characterization far from equilibrium.

Analysis of our operando XRD data allowed a quantitative correlation of oxygen vacancy non-stoichiometry to cathodic cell current. This demonstrates that by electrochemical stimulus, we could feasibly modify the concentration of oxygen vacancy without change in gas atmosphere and essentially control the chemical composition $\mathrm{CeO}_{2-x}$ in a steady state. Another implication lies in the electromechanical coupling. For ceria and ceria-based energy conversion and storage devices, the operation under non-equilibrium condition could introduce substantial chemical expansion/contraction of the material, to which one needs to pay attention for mechanical durability concerns. The same applies to other fluoritestructured materials.

When the electrochemical potential is large enough to drive ceria into a miscibility gap, phase separation occurs and a mixture of $\alpha$ and $\alpha^{\prime}$ cubic phases is observed. Relative intensities of the two phases suggest that the phase transition occurs inside each nanocrystal of ceria. An interesting find is that the phase transition behavior from $\alpha$ to $\alpha^{\prime}$ induced by electrochemical potentials differs from that under thermal equilibrium condition. Depending on the magnitude of cell current, phase transition completes at different stoichiometric states even at the same temperature. Such difference indicates a relative shift of the electrochemical free energy curves of the two phases regardless of a retained temperature. This phase transition achieved by electrochemical potential opens up new possibilities in using potential to control the phase of a functioning oxide materials. However, a more quantitative understanding of the phase change mechanism at steady state is required and will be the focus of our future work.

\section{EXPERIMENTAL SECTION}

In Situ Setup. The cell was mounted on a specially designed sample holder with an X-ray transparent dome to insure a constant atmosphere during cell operation. The dome was air-cooled when the temperature reached over $200{ }^{\circ} \mathrm{C}$.

For the XRD and XAS experiments, a WT of $650{ }^{\circ} \mathrm{C}$ was set at the heating stage. However, because of temperature gradient from the bottom to the top of the cell, the probed region experienced a lower
WT of $610-615{ }^{\circ} \mathrm{C}$ (see Supporting Information for details). The cell was exposed to a controlled atmosphere composing 20 Torr of $\mathrm{H}_{2} \mathrm{O}$ and 20 Torr of $\mathrm{H}_{2}$. Applied voltage varied from +1.2 to $-1.2 \mathrm{~V}$ for $\mathrm{H}_{2} \mathrm{O}$ splitting and $\mathrm{H}_{2}$ oxidation on the ceria electrode, respectively.

Micro-XRD. Powder X-ray microdiffraction experiments were conducted at beamline 12.3.2 of the Advanced Light Source of the Lawrence Berkeley National Laboratory. A high-brilliance X-ray beam $(6.5 \mathrm{keV})$ was focused down to about $4 \mu \mathrm{m} \times 10 \mu \mathrm{m}$ using a pair of Kirkpatrick-Baez mirrors. ${ }^{36,37}$ Diffraction patterns were collected using a two-dimensional DECTRIS Pilatus $1 \mathrm{M}$ X-ray detector, positioned at $70^{\circ}$ with respect to the incident beam. The sample was tilted to an angle of $30^{\circ}$ relative to the incident beam (reflection geometry). The distance from the sample to the center of the detector was $\sim 138 \mathrm{~mm}$. An exposure time of $160 \mathrm{~s}$ per pattern was used. The sample-detector distance, the center channel of the detector, and the tilt of the detector relative to the sample surface were calibrated using a Laue pattern obtain from a strain-free $\mathrm{Si}$ single-crystal. Data processing is detailed in Supporting Information.

Micro-XAS. Hard X-ray microprobe measurements were performed at beamline 10.3.2 of the Advanced Light Source of the Lawrence Berkeley National Laboratory. ${ }^{38}$ This beamline includes a pair of focusing Kirkpatrick-Baez mirrors and a Si 111 two-crystal monochromator which forms the X-ray micro-beam. A Canberra 7element UltraLeGe solid-state detector records the fluorescence from the sample.

A series of X-ray fluorescence maps were collected at the ceria/Au electrode. The $\mathrm{Ce}^{3+}$ and $\mathrm{Ce}^{4+}$ chemical species were identified by mapping the sample using several incident energies around the Ce emission edge: $5710 \mathrm{eV}$ (pre-edge), $5727 \mathrm{eV}$ (mostly $\mathrm{Ce}^{3+}$ ), 5738.4 $\mathrm{eV}\left(\right.$ mostly $\left.\mathrm{Ce}^{4+}\right)$, and $5747 \mathrm{eV}$ (post-edge). Energy calibration was such that the first peak of the XANES of $\mathrm{CeO}_{2}$ was defined to be at $5730.39 \mathrm{eV}$. An additional map was collected above the $\mathrm{Au} \mathrm{L}_{3}$ edge in order to locate the $\mathrm{Au}$ region.

XAS data were acquired in fluorescence mode using the quick XAS method, by slewing the monochromator continuously and at a constant speed from low to high energies as the X-ray absorption data got recorded. This mode allowed the collection of spectra in the $5620-5940 \mathrm{eV}$ range with $2 \mathrm{eV}$ step size $(0.3 \mathrm{eV}$ in the XANES region) and $\sim 5 \mathrm{~s} /$ point summed over 30 sweeps $(0.81 \mathrm{~s} /$ point in the XANES region). Each sweep from low to high energy took about 1 min; so a scan of 30 sweeps took half an hour. The energy scale of each sweep was repeatable to $\sim 0.1 \mathrm{eV}$. Ten scans were acquired at each spot of interest in order to improve the signal-to-noise ratio. For simplicity, a linear combination of two models were used in data fitting. The first model is the fluorite-type cubic structure of ceria with the $\mathrm{Ce}$ atoms surrounded by eight oxygen neighbors; while the second one has the same structure consisting of four oxygen neighbors and four vacancies surrounding the $\mathrm{Ce}$ atoms. Further details can be found in Supporting Information.

\section{ASSOCIATED CONTENT}

\section{Supporting Information}

The Supporting Information is available free of charge at https://pubs.acs.org/doi/10.1021/acsami.0c08284.

Anton-Paar DHS heater during operando measurement, current-voltage profile, simulation of powder diffraction patterns at $611^{\circ} \mathrm{C}$, relative intensity of $\alpha$ and $\alpha^{\prime}$ phases as a function of $2-x$, coherent domain size of the two phases as a function of respective intensity, evolution of relative intensity and $2-x$ value of $\alpha^{\prime}$ as a function of WT, position limits of $\alpha^{\prime}$ phase, powder diffraction patterns, calibration of WT, oxygen non-stoichiometry calculation, refinement results for $611{ }^{\circ} \mathrm{C}$ at $\mathrm{OCV}$, applied potentials of $+0.3,+0.6,+0.9$, and $+1.2 \mathrm{~V}$, refinement results for an applied potential of $+1.2 \mathrm{~V}$ at 511-661 ${ }^{\circ} \mathrm{C}$, data processing from XAS measurements, 
$k^{2}$-weighted $\mathrm{Ce}_{3}$ EXAFS spectra, and corresponding Fourier transform results (PDF)

\section{AUTHOR INFORMATION}

\section{Corresponding Authors}

Bryan W. Eichhorn - Department of Chemistry and Biochemistry, University of Maryland, College Park, Maryland 20742, United States; (1) orcid.org/0000-0001-9161-1920; Email: eichhorn@umd.edu

Zhi Liu - School of Physical Science and Technology, ShanghaiTech University, Shanghai 201210, China; Advanced Light Source, Lawrence Berkeley National Laboratory, Berkeley, California 94720, United States; State Key Laboratory of Functional Materials for Informatics, Shanghai Institute of Microsystem and Information Technology, Chinese Academy of Sciences, Shanghai 200050, China; 이이.org/0000-00028973-6561; Email: liuzhi@shanghaitech.edu.cn

\section{Authors}

Catherine Dejoie - European Synchrotron Radiation Facility, Grenoble Cedex 9 38043, France; Advanced Light Source, Lawrence Berkeley National Laboratory, Berkeley, California 94720, United States

Yi Yu - School of Physical Science and Technology, ShanghaiTech University, Shanghai 201210, China; Department of Chemistry and Biochemistry, University of Maryland, College Park, Maryland 20742, United States; (1) orcid.org/0000-0003-1667-5187

Fabiano Bernardi - Programa de Pós-Graduação em Fisica, Instituto de Física, Universidade Federal do Rio Grande do Sul (UFRGS), Porto Alegre 91501-970, Rio Grande do Sul, Brazil; Advanced Light Source, Lawrence Berkeley National Laboratory, Berkeley, California 94720, United States; (1) orcid.org/0000-0001-6817-6860

Nobumichi Tamura - Advanced Light Source, Lawrence Berkeley National Laboratory, Berkeley, California 94720, United States

Martin Kunz - Advanced Light Source, Lawrence Berkeley National Laboratory, Berkeley, California 94720, United States

Matthew A. Marcus - Advanced Light Source, Lawrence Berkeley National Laboratory, Berkeley, California 94720, United States

Yi-Lin Huang - Department of Chemistry and Biochemistry, University of Maryland, College Park, Maryland 20742, United States; (1) orcid.org/0000-0002-1886-3352

Chunjuan Zhang - Department of Chemistry and Biochemistry, University of Maryland, College Park, Maryland 20742, United States

Complete contact information is available at:

https://pubs.acs.org/10.1021/acsami.0c08284

\section{Author Contributions}

${ }^{\mathrm{II}}$ C.D. and Y.Y. contributed equally to this article.

\section{Author Contributions}

C.D., F.B., and Z.L. conceived the experiment. Y.Y. and C.Z. fabricated the SOCs. C.D., N.T., M.K., F.B., M.A.M., and Z.L. carried out the measurements. C.D., N.T., and M.K. performed the XRD analysis and M.A.M., Y.L.H., and F.B. interpreted the XAS and electrochemical results. B.W.E. and Z.L. supervised the project. Y.Y. and C.D. drafted the manuscript. All authors contributed to writing the manuscript.

\section{Notes}

The authors declare no competing financial interest.

\section{ACKNOWLEDGMENTS}

We thank the Office of Naval Research for financial support and the Maryland Energy Innovation Institute for experimental support. F.B. thanks the $\mathrm{CNPq}$ for the research grant. This research used beamline 10.3.2 and 12.3.2 of the Advanced Light Source, supported by the Director, Office of Science, Office of Basic Energy Sciences, US Department of Energy under Contract no. DE-AC02-05CH11231. We appreciate support from the National Natural Science Foundation of China (no. 11227902, no. 21991152 and no. 21832004) and Shanghai-XFEL Beamline Project (SBP) (no. 31011505505885920161A2101001). We sincerely appreciate the anonymous reviewer for the helpful comments and valuable suggestions.

\section{REFERENCES}

(1) Trovarelli, A. Catalytic Properties of Ceria and $\mathrm{CeO}_{2}$-Containing Materials. Catal. Rev. 1996, 38, 439-520.

(2) Fu, Q.; Saltsburg, H.; Flytzani-Stephanopoulos, M. Active Nonmetallic Au and Pt Species on Ceria-Based Water-Gas Shift Catalysts. Science 2003, 301, 935-938.

(3) Park, S.; Vohs, J. M.; Gorte, R. J. Direct Oxidation of Hydrocarbons in a Solid-Oxide Fuel Cell. Nature 2000, 404, 265267.

(4) Mogensen, M.; Sammes, N. M.; Tompsett, G. A. Physical, Chemical and Electrochemical Properties of Pure and Doped Ceria. Solid State Ionics 2000, 129, 63-94.

(5) Chueh, W. C.; Hao, Y.; Jung, W.; Haile, S. M. High Electrochemical Activity of the Oxide Phase in Model Ceria-Pt and Ceria-Ni Composite Anodes. Nat. Mater. 2011, 11, 155-161.

(6) Tuller, H. L.; Bishop, S. R. Point Defects in Oxides: Tailoring Materials through Defect Engineering. Annu. Rev. Mater. Res. 2011, 41, 369-398.

(7) Stoukides, M. Solid-Electrolyte Membrane Reactors: Current Experience and Future Outlook. Catal. Rev. 2000, 42, 1-70.

(8) Sun, C.; Li, H.; Chen, L. Nanostructured Ceria-Based Materials: Synthesis, Properties, and Applications. Energy Environ. Sci. 2012, 5, 8475-8505.

(9) Schmitt, R.; Spring, J.; Korobko, R.; Rupp, J. L. M. Design of Oxygen Vacancy Configuration for Memristive Systems. ACS Nano 2017, 11, 8881-8891.

(10) Montini, T.; Melchionna, M.; Monai, M.; Fornasiero, P. Fundamentals and Catalytic Applications of $\mathrm{CeO}_{2}$-Based Materials. Chem. Rev. 2016, 116, 5987-6041.

(11) Alessandro, T.; Paolo, F. Catalysis by Ceria and Related Materials, 2nd ed.; World Scientific Publishing Company; 2013.

(12) Chueh, W. C.; Falter, C.; Abbott, M.; Scipio, D.; Furler, P.; Haile, S. M.; Steinfeld, A. High-Flux Solar-Driven Thermochemical Dissociation of $\mathrm{CO}_{2}$ and $\mathrm{H}_{2} \mathrm{O}$ Using Nonstoichiometric Ceria. Science 2010, 330, 1797-1801.

(13) Bishop, S. R.; Marrocchelli, D.; Chatzichristodoulou, C.; Perry, N. H.; Mogensen, M. B.; Tuller, H. L.; Wachsman, E. D. Chemical Expansion: Implications for Electrochemical Energy Storage and Conversion Devices. Annu. Rev. Mater. Res. 2014, 44, 205-239.

(14) Swallow, J. G.; Kim, J. J.; Maloney, J. M.; Chen, D.; Smith, J. F.; Bishop, S. R.; Tuller, H. L.; Van Vliet, K. J. Dynamic Chemical Expansion of Thin-Film Non-Stoichiometric Oxides at Extreme Temperatures. Nat. Mater. 2017, 16, 749-754.

(15) Li, Y.; Chueh, W. C. Electrochemical and Chemical Insertion for Energy Transformation and Switching. Annu. Rev. Mater. Res. 2018, 48, 137-165.

(16) Strachan, J. P.; Pickett, M. D.; Yang, J. J.; Aloni, S.; David Kilcoyne, A. L.; Medeiros-Ribeiro, G.; Stanley Williams, R. Direct 
Identification of the Conducting Channels in a Functioning Memristive Device. Adv. Mater. 2010, 22, 3573-3577.

(17) Tang, M.; Carter, W. C.; Chiang, Y.-M. Electrochemically Driven Phase Transitions in Insertion Electrodes for Lithium-Ion Batteries: Examples in Lithium Metal Phosphate Olivines. Annu. Rev. Mater. Res. 2010, 40, 501-529.

(18) Xiong, F.; Wang, H.; Liu, X.; Sun, J.; Brongersma, M.; Pop, E.; Cui, Y. Li Intercalation in $\mathrm{MoS}_{2}:$ In Situ Observation of Its Dynamics and Tuning Optical and Electrical Properties. Nano Lett. 2015, 15, 6777-6784.

(19) Zhang, C.; Grass, M. E.; McDaniel, A. H.; DeCaluwe, S. C.; Gabaly, F. E.; Liu, Z.; McCarty, K. F.; Farrow, R. L.; Linne, M. A.; Hussain, Z.; Jackson, G. S.; Bluhm, H.; Eichhorn, B. W. Measuring Fundamental Properties in Operating Solid Oxide Electrochemical Cells by Using in situ X-Ray Photoelectron Spectroscopy. Nat. Mater. 2010, 9, 944-949.

(20) Zhang, C.; Grass, M. E.; Yu, Y.; Gaskell, K. J.; DeCaluwe, S. C.; Chang, R.; Jackson, G. S.; Hussain, Z.; Bluhm, H.; Eichhorn, B. W.; Liu, Z. Multielement Activity Mapping and Potential Mapping in Solid Oxide Electrochemical Cells through the Use of Operando XPS. ACS Catal. 2012, 2, 2297-2304.

(21) Zhang, C.; Yu, Y.; Grass, M. E.; Dejoie, C.; Ding, W.; Gaskell, K.; Jabeen, N.; Hong, Y. P.; Shavorskiy, A.; Bluhm, H.; Li, W.-X.; Jackson, G. S.; Hussain, Z.; Liu, Z.; Eichhorn, B. W. Mechanistic Studies of Water Electrolysis and Hydrogen Electro-Oxidation on High Temperature Ceria-Based Solid Oxide Electrochemical Cells. J. Am. Chem. Soc. 2013, 135, 11572-11579.

(22) Yu, Y.; Mao, B.; Geller, A.; Chang, R.; Gaskell, K.; Liu, Z.; Eichhorn, B. W. $\mathrm{CO}_{2}$ Activation and Carbonate Intermediates: An Operando AP-XPS Study of $\mathrm{CO}_{2}$ Electrolysis Reactions on Solid Oxide Electrochemical Cells. Phys. Chem. Chem. Phys. 2014, 16, 11633-11639.

(23) Lu, Q.; Yildiz, B. Voltage-Controlled Topotactic Phase Transition in Thin-Film $\mathrm{SrCoO}_{\mathrm{x}}$ Monitored by In Situ X-Ray Diffraction. Nano Lett. 2016, 16, 1186-1193.

(24) Adachi, G.-y.; Imanaka, N. The Binary Rare Earth Oxides. Chem. Rev. 1998, 98, 1479-1514.

(25) Jiang, Y.; Adams, J. B.; van Schilfgaarde, M. Density-Functional Calculation of $\mathrm{CeO}_{2}$ Surfaces and Prediction of Effects of Oxygen Partial Pressure and Temperature on Stabilities. J. Chem. Phys. 2005, 123, 064701.

(26) Fabris, S.; Vicario, G.; Balducci, G.; de Gironcoli, S.; Baroni, S. Electronic and Atomistic Structures of Clean and Reduced Ceria Surfaces. J. Phys. Chem. B 2005, 109, 22860-22867.

(27) Nolan, M.; Parker, S. C.; Watson, G. W. $\mathrm{CeO}_{2}$ Catalysed Conversion of $\mathrm{CO}, \mathrm{NO}_{2}$ and $\mathrm{NO}$ from First Principles Energetics. Phys. Chem. Chem. Phys. 2006, 8, 216-218.

(28) Chen, H.-T.; Choi, Y. M.; Liu, M.; Lin, M. C. A Theoretical Study of Surface Reduction Mechanisms of $\mathrm{CeO}_{2}(111)$ and (110) by $\mathrm{H}_{2}$. ChemPhysChem 2007, 8, 849-855.

(29) Chueh, W. C.; McDaniel, A. H.; Grass, M. E.; Hao, Y.; Jabeen, N.; Liu, Z.; Haile, S. M.; McCarty, K. F.; Bluhm, H.; El Gabaly, F. Highly Enhanced Concentration and Stability of Reactive $\mathrm{Ce}^{3+}$ on Doped $\mathrm{CeO}_{2}$ Surface Revealed in Operando. Chem. Mater. 2012, 24, $1876-1882$.

(30) Bevan, D. J. M. Ordered Intermediate Phases in the System $\mathrm{CeO}_{2}-\mathrm{Ce}_{2} \mathrm{O}_{3}$. J. Inorg. Nucl. Chem. 1955, 1, 49-59.

(31) Kim, D.-J. Lattice Parameters, Ionic Conductivities, and Solubility Limits in Fluorite-Structure $\mathrm{MO}_{2}$ Oxide $\left[\mathrm{M}=\mathrm{Hf}^{++}, \mathrm{Zr}^{4+}\right.$, $\left.\mathrm{Ce}^{4+}, \mathrm{Th}^{4+}, \mathrm{U}^{4+}\right]$ Solid Solutions. J. Am. Ceram. Soc. 1989, 72, 14151421.

(32) Riess, I.; Ricken, M.; Nölting, J. On the Specific Heat of Nonstoichiometric Ceria. J. Solid State Chem. 1985, 57, 314-322.

(33) Jeong, J.; Aetukuri, N.; Graf, T.; Schladt, T. D.; Samant, M. G.; Parkin, S. S. P. Suppression of Metal-Insulator Transition in $\mathrm{VO}_{2}$ by Electric Field-Induced Oxygen Vacancy Formation. Science 2013, 339, 1402-1405.
(34) Chen, D.; Tuller, H. L. Voltage-Controlled Nonstoichiometry in Oxide Thin Films: $\operatorname{Pr}_{0.1} \mathrm{Ce}_{0.9} \mathrm{O}_{2-\delta}$ Case Study. Adv. Funct. Mater. 2014, 24, 7638-7644.

(35) Bard, A. J.; Faulkner, L. R. Electrochemical Methods: Fundamentals and Applications; John Wiley \& Sons: New York, 2000.

(36) Tamura, N.; MacDowell, A. A.; Spolenak, R.; Valek, B. C.; Bravman, J. C.; Brown, W. L.; Celestre, R. S.; Padmore, H. A.; Batterman, B. W.; Patel, J. R. Scanning X-Ray Microdiffraction with Submicrometer White Beam for Strain/Stress and Orientation Mapping in Thin Films. J. Synchrotron Radiat. 2003, 10, 137-143.

(37) Kunz, M.; Tamura, N.; Chen, K.; MacDowell, A. A.; Celestre, R. S.; Church, M. M.; Fakra, S.; Domning, E. E.; Glossinger, J. M.; Kirschman, J. L.; Morrison, G. Y.; Plate, D. W.; Smith, B. V.; Warwick, T.; Yashchuk, V. V.; Padmore, H. A.; Ustundag, E. A Dedicated Superbend X-Ray Microdiffraction Beamline for Materials, Geo-, and Environmental Sciences at the Advanced Light Source. Rev. Sci. Instrum. 2009, 80, 035108.

(38) Marcus, M. A.; MacDowell, A. A.; Celestre, R.; Manceau, A.; Miller, T.; Padmore, H. A.; Sublett, R. E. Beamline 10.3.2 at ALS: A Hard X-Ray Microprobe for Environmental and Materials Sciences. J. Synchrotron Radiat. 2004, 11, 239-247. 